Document downloaded from:

http://hdl.handle.net/10251/169063

This paper must be cited as:

Bonet Solves, JA.; Galbis, A. (2020). Invariant subspaces of the integration operators on Hörmander algebras and Korenblum type spaces. Integral Equations and Operator Theory. 92(4):1-13. https://doi.org/10.1007/s00020-020-02593-6

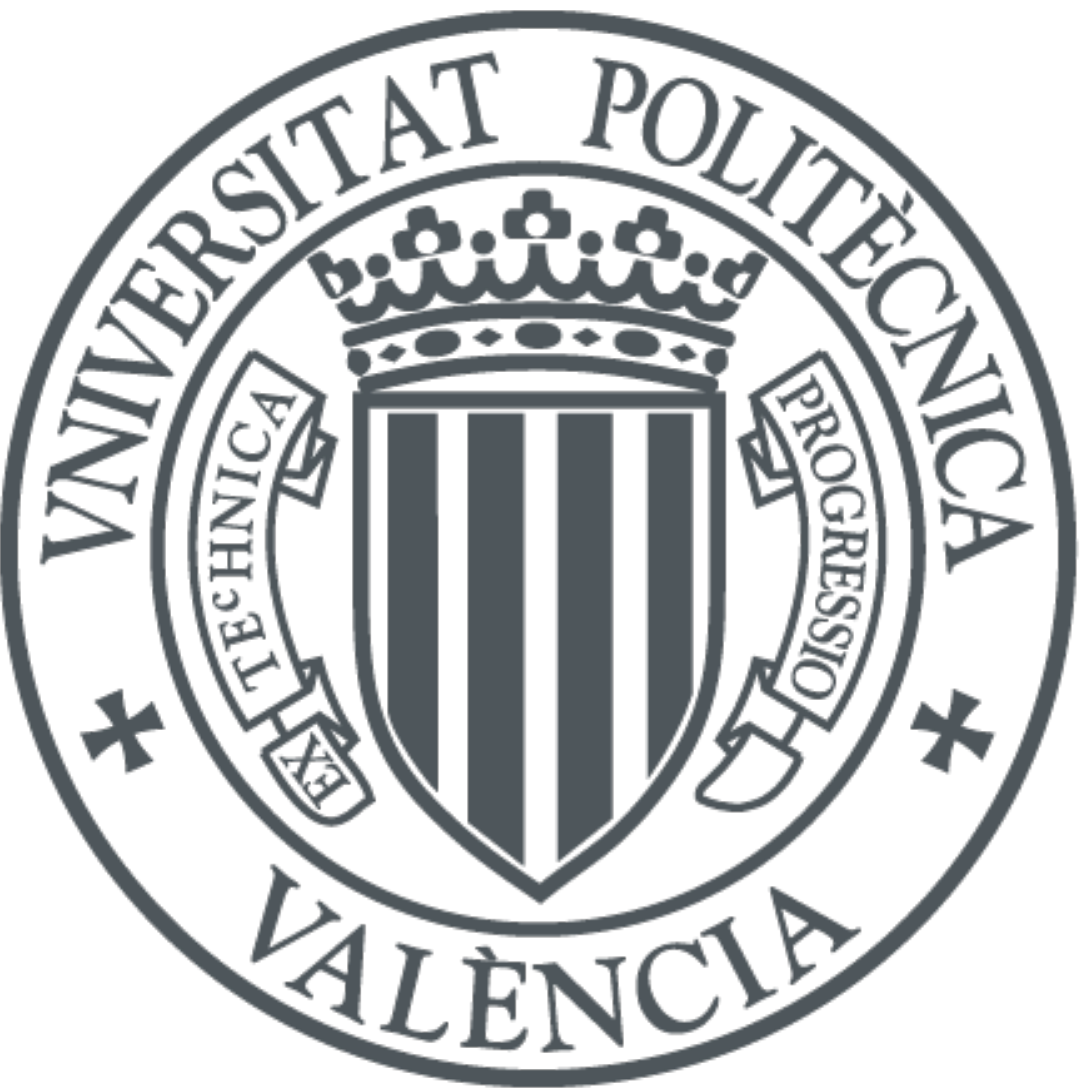

The final publication is available at

https://doi.org/10.1007/s00020-020-02593-6

Copyright Springer-Verlag

Additional Information 


\title{
Invariant subspaces of the integration operators on Hörmander algebras and Korenblum type spaces
}

\author{
José Bonet and Antonio Galbis
}

\begin{abstract}
We describe the proper closed invariant subspaces of the integration operator when it acts continuously on countable intersections and countable unions of weighted Banach spaces of holomorphic functions on the unit disc or the complex plane. Applications are given to the case of Korenblum type spaces and Hörmander algebras of entire functions.
\end{abstract}

\section{Introduction.}

Let $G$ be the open unit disc $\mathbb{D}$ or the whole complex plane $\mathbb{C}$. We denote by $H(G)$ the Fréchet space of holomorphic functions on $G$, endowed with the topology of uniform convergence on compact subsets of $G$. A space $E$ of holomorphic functions on the domain $G$ is a Hausdorff locally convex space that is a subset of $H(G)$, such that the inclusion map $E \subset H(G)$ is continuous and $E$ contains the polynomials. If $E$ is a Fréchet space or a countable inductive limit of Banach spaces, by the closed graph theorem, the inclusion map $E \subset H(G)$ is continuous if and only if the point evaluations $E \rightarrow \mathbb{C}, f \rightarrow f(z)$ at all the points $z \in G$ are continuous on $E$. We are mainly interested in the case when the polynomials are dense in $E$. In this case then $E$ is separable.

Banach spaces of holomorphic functions on the unit disc $\mathbb{D}$ and on the complex plane $\mathbb{C}$ have been thoroughly investigated. We refer the reader for example to the books [23, 34] and [35. Hörmander algebras of entire functions [1], [10], [11, [17, [28, as well as Koremblum space and other intersections and unions of growth Banach spaces of holomorphic functions on the unit disc [6], [18, [23], are natural examples of (locally convex) spaces of holomorphic functions. Vogt 32] proved that there are Fréchet spaces $E$ which are contained in $H(G)$ such that the inclusion $E \subset H(G)$ is not continuous.

In this note we study the set of proper closed invariant subspaces of the integration operator

$$
J f(z):=\int_{0}^{z} f(\zeta) d \zeta, \quad z \in G, \quad f \in H(G),
$$

when it acts continuously on Fréchet spaces or countable inductive limits of Banach spaces (called (LB)-spaces) E, which appear as countable intersections or unions of weighted Banach spaces of holomorphic functions on the unit disc or the complex plane. Recall that a subspace $M$ of a locally convex space $E$ is called invariant of a continuous linear operator $T: E \rightarrow E$ if $T(M) \subset M$. Our main results are Theorem4.1 and its Corollary 4.2,

2020 Mathematics Subject Classification. Primary: 47A15, 47B38, secondary: 46A04, 46A13, 46E10.

Key words and phrases. Integration operator, invariant subspaces, weighted spaces of holomorphic functions, Fréchet spaces, (LB)-spaces

This article is an improved version of the paper [16], which appeared in Arxiv, reference 2003.13573 
which describe the invariant subspaces when the integration operator acts on Korenblum type spaces, and Theorem [5.1, which explains the situation in case of some Hörmander algebras of entire functions. The proofs of these results depend on some abstract Theorems 3.2 and 3.3 and they rely heavily on Theorems 3.8 and 3.16 due to Abanin and Tien [4]. A different method permits us to handle the (LB)-algebra of entire functions of exponential type in Theorem 5.3. An open question about the invariant subspaces of the integration operator on certain Hörmander algebras is mentioned in Remark 5.4.

Abanin and Tien describe in [4 the closed invariant subspaces of the integration operator on various scales of weighted Banach spaces of holomorphic functions. As mentioned above, some of their results are very important for our theorems below. We refer the reader to the introduction of 4 for classical results about invariant subspaces of the integration operators and more recent ones in [7, [19] and [20]. The continuity of the integration operator on weighted Banach spaces of holomorphic functions was investigated by Harutyunyan and Lusky [22]; see also [2] and [5]. Other aspects, like spectrum and ergodic or dynamical properties, were considered by Beltrán, Fernández and the first author in [8]. Similar questions for operators defined on Hörmander algebras were investigated in [9].

Our notation for functional analysis, in particular for locally convex spaces, Fréchet spaces and (LB)-spaces, is standard. We refer the reader to [12, 24], 29] and [31]. If $E$ is a locally convex space, its topological dual is denoted by $E^{\prime}$. The linear span of a subset $A$ of $E$ is denoted by $\operatorname{span}(A)$. The closure of a subset $A$ in $E$ is denoted by $\bar{A}$, and $\bar{A}^{E}$ in case the space in which the closure is taken must be emphasized. A subspace $M$ of $E$ is called proper if $\{0\} \neq M \neq E$. In what follows, we set $\mathbb{N}_{0}:=\mathbb{N} \cup\{0\}$.

\section{Results about subspaces of Fréchet or (LB)-spaces.}

Lemma 2.1 Let $X:=\operatorname{proj}_{n} X_{n}$ be a Fréchet space such that $X=\bigcap_{n \in \mathbb{N}} X_{n}$ with each $\left(X_{n},\|\cdot\|_{n}\right)$ a Banach space. Moreover, it is assumed that $X$ is dense in $X_{n}$ and that $X_{n+1} \subseteq X_{n}$ with a continuous inclusion for each $n \in \mathbb{N}$. Let $M$ be a subspace of $X$. Then

(i) $\bar{M}^{X}=\bigcap_{n \geq 1} \bar{M}^{X_{n}}$.

(ii) If $M$ is proper and closed, then there is $n(0) \in \mathbb{N}$ such that $\bar{M}^{X_{n}}$ is proper in $X_{n}$ for each $n \geq n(0)$.

Proof. (i) Since the inclusions $X \subset X_{n+1} \subset X_{n}$ are continuous for each $n \in \mathbb{N}$, we clearly have $\bar{M}^{X} \subset \bigcap_{n \geq m} \bar{M}^{X_{n}}=\bigcap_{n \geq 1} \bar{M}^{X_{n}}$ for each $m \in \mathbb{N}$. Now, given $x \in \bigcap_{n \geq 1} \bar{M}^{X_{n}}$, we have $x \in \bigcap_{n \geq 1} X_{n}=X$. Moreover, for each $n \in \mathbb{N}$ there is $y(n) \in M$ such that $\|x-y(n)\|_{n}<1 / n$. Then $x=\lim _{n \rightarrow \infty} y(n)$ in $X$ and $x \in \bar{M}^{X}$.

(ii) First of all, since $\{0\} \neq M$, we get $\{0\} \neq \bar{M}^{X_{n}}$ for each $n \in \mathbb{N}$. Proceeding by contradiction, suppose that there is an increasing sequence $(n(k))_{k}$ of natural numbers such that $\bar{M}^{X_{n(k)}}=X_{n(k)}$ for each $k \in \mathbb{N}$. Since $X:=\operatorname{proj}_{k} X_{n(k)}$, we could apply part (i) to conclude $\bar{M}^{X}=X$. Since $M$ is closed by assumption, we have $M=X$, and $M$ would not be a proper subspace.

Lemma 2.2 Let $X=\operatorname{ind}_{n} X_{n}$ be an (LB)-space with increasing union of Banach spaces $X=\bigcup_{n \in \mathbb{N}} X_{n}$. If $M$ is a proper closed subspace of $X$, then there is $n(0) \in \mathbb{N}$ such that $M \cap X_{n}$ is a proper closed subspace of $X_{n}$ for each $n \geq n(0)$. 
Proof. Since the inclusion $X_{n} \subset X$ is continuous for each $n \in \mathbb{N}$, the set $M \cap X_{n}$ is closed in $X_{n}$ for each $n \in \mathbb{N}$. As $M$ is proper, there is $x \in M, x \neq 0$. There is $n(1) \in \mathbb{N}$ such that $x \in M \cap X_{n(1)}$. On the other hand, since $M \neq X$, there is $y \in X \backslash M$. Select $n(0) \geq n(1)$ such that $y \in X_{n(0)}$. Then, if $n \geq n(0)$, we have $x \in M \cap X_{n}$ and $y \in X_{n} \backslash M$. Thus, $M \cap X_{n}$ is a proper subspace of $X_{n}$

\section{Abstract results about invariant subspaces of the integra- tion operator on spaces of holomorphic functions.}

Our first Lemma is stated in page 412 of [4. We include a proof for the sake of completeness, because it is very relevant in our considerations below. As in [4], given a space of holomorphic functions $E$ on the open unit disc $\mathbb{D}$ or the complex plane $\mathbb{C}$, and $N \in \mathbb{N}$, we set

$$
A_{N}^{E}:=\left\{f \in E \mid f^{(j)}(0)=0,0 \leq j<N\right\} .
$$

Lemma 3.1 Let $E$ be a space of holomorphic functions on the open domain $G=\mathbb{D}$ or $G=\mathbb{C}$, such that the polynomials are dense in $E$. For each $N \in \mathbb{N}$ we have

$$
A_{N}^{E}=\overline{\operatorname{span}\left(\left\{z^{j} \mid j \geq N\right\}\right)} \text {. }
$$

Proof. Fix $n \in \mathbb{N}$. Since the inclusion $E \subset H(G)$ is continuous, the evaluations $E \rightarrow$ $\mathbb{C}, f \rightarrow f^{(j)}(0)$ are continuous for each $j \in \mathbb{N}$ and the set $A_{N}^{E}$ is closed. Moreover, it clearly contains $z^{j}$ for each $j \geq N$. Hence, $\overline{\operatorname{span}\left(\left\{z^{j} \mid j \geq N\right\}\right)} \subset A_{N}^{E}$.

The Taylor expansion at the origin of a holomorphic function $f \in H(G)$ is denoted by $f(z)=\sum_{j=0}^{\infty} a_{j}(f) z^{j}$, for each $z \in G$. Fix $f \in A_{N}^{E}$. Since the polynomials are dense in $E$ by assumption, there is a sequence of polynomials $\left(g_{k}\right)_{k}$ such that $\lim _{k \rightarrow \infty} g_{k}=f$ in $E$. This implies that $\lim _{k \rightarrow \infty} g_{k}=f$ in $H(G)$. Therefore, $\lim _{k \rightarrow \infty} \sum_{j=0}^{N-1} a_{j}\left(g_{k}\right) z^{j}=0$ in $H(G)$. The span of $\left\{1, z, \ldots, z^{N-1}\right\}$ is finite dimensional, hence $\lim _{k \rightarrow \infty} \sum_{j=0}^{N-1} a_{j}\left(g_{k}\right) z^{j}=0$ in $E$. Consequently, $\left(g_{k}-\sum_{j=0}^{N-1} a_{j}\left(g_{k}\right) z^{j}\right)_{k}$ is a sequence of elements of $\operatorname{span}\left(\left\{z^{j} \mid j \geq N\right\}\right)$ which converges in the topology of $E$ to $f$. Thus, $f \in \overline{\operatorname{span}\left(\left\{z^{j} \mid j \geq N\right\}\right)}$.

Theorem 3.2 Let $F:=\operatorname{proj}_{n} F_{n}$ be a Fréchet space such that $F=\bigcap_{n \in \mathbb{N}} F_{n}$ with each $F_{n}$ a Banach space of holomorphic functions on the open domain $G=\mathbb{D}$ or $G=\mathbb{C}$, such that the polynomials are contained in $F$ and dense in $F_{n}$ for each $n \in \mathbb{N}$.

Assume that the integration operator $J: F_{n} \rightarrow F_{n}$ is continuous for each $n \in \mathbb{N}$, and that, for each $n \in \mathbb{N}$, every proper closed invariant subspace of $J$ on $F_{n}$ is of the form $A_{K}^{F_{n}}$ for some $K \in \mathbb{N}$. Then

(i) $J: F \rightarrow F$ is continuous, and

(ii) Every proper closed invariant subspace for $J$ on $F$ is of the form

$$
A_{K}^{F}=\left\{f \in F \mid f^{(j)}(0)=0,0 \leq j<K\right\}
$$

for some $K \in \mathbb{N}$.

Proof. (i) The continuity of $J: F_{n} \rightarrow F_{n}$ for each $n \in \mathbb{N}$ clearly implies $J(F) \subset F$ and that $J: F \rightarrow F$ is continuous, because $F$ is the projective limit of the sequence of Banach spaces $\left(F_{n}\right)_{n}$. 
(ii) It is easy to see that $A_{K}^{F}$ is a closed invariant subspace of the integration operator $J$ on $F$; see for example Lemma 3.1. Since $1 \notin A_{K}^{F}$ and $z^{K} \in A_{K}^{F}$, it follows $A_{K}^{F}$ is a proper subspace of $E$.

Now, let $M$ be a proper closed subspace of $F$ which is invariant for the integration operator $J$. By Lemma 2.1 (ii) there is $n(0) \in \mathbb{N}$ such that $\bar{M}^{F_{n}}$ is proper (and closed) in $F_{n}$ for each $n \geq n(0)$. Moreover, it is invariant for $J$, since

$$
J\left(\bar{M}^{F_{n}}\right) \subset \overline{J(M)}^{F_{n}} \subset \bar{M}^{F_{n}} .
$$

By assumption, for each $n \geq n(0)$, there is $k(n) \in \mathbb{N}$ such that

$$
\bar{M}^{F_{n}}=A_{k(n)}^{F_{n}}=\overline{\operatorname{span}\left(\left\{z^{j} \mid j \geq k(n)\right\}\right)} F_{n} .
$$

Take, for each $n \in \mathbb{N}, k(n)$ as the minimum of those $j^{\prime} s$ such that $z^{j} \in \bar{M}^{F_{n}}$. Clearly $1 \leq k(n)$ for each $n \geq n(0)$. This selection implies, in particular, that $z^{k(n)-1} \notin \bar{M}^{F_{n}}$. Since $\bar{M}^{F_{n+1}} \subset \bar{M}^{F_{n}}$ for each $n \in \mathbb{N}$, we have $1 \leq k(n(0)) \leq k(n) \leq k(n+1)$ for each $n \geq n(0)$.

We claim that the sequence $(k(n))_{n}$ of natural numbers must be bounded. If this is not the case, $\lim _{n \rightarrow \infty} k(n)=\infty$. For each $n \geq n(0)$ we have

$$
M \subset \bar{M}^{F_{n}}=\overline{\operatorname{span}\left(\left\{z^{j} \mid j \geq k(n)\right\}\right)}{ }^{F_{n}} \subset\left\{f \in H(G) \mid f^{(j)}(0)=0,0 \leq j<k(n)\right\} .
$$

Therefore, the Taylor coefficients $\left(a_{j}(f)\right)_{j=0}^{\infty}$ of each $f \in M$ must vanish; that is, $M=\{0\}$, which is a contradiction, as $M$ is proper.

As a consequence of the claim that we have just proved, there are $K \in \mathbb{N}$ and $n(1) \geq$ $n(0)$ such that $k(n)=K$ for each $n \geq n(1)$. This implies

$$
\bar{M}^{F_{n}}=\left\{f \in F_{n} \mid f^{(j)}(0)=0,0 \leq j<K\right\}
$$

for each $n \geq n(1)$. Thus, we may apply Lemma 2.1(i) to get

$$
M=\bigcap_{n \geq n(1)} \bar{M}^{F_{n}}=\left\{f \in F \mid f^{(j)}(0)=0,0 \leq j<K\right\} .
$$

This completes the proof.

Theorem 3.3 Let $E=\operatorname{ind}_{n} E_{n}$ be an (LB)-space which is the increasing union of Banach spaces $E_{n}$ of holomorphic functions on the open domain $G=\mathbb{D}$ or $G=\mathbb{C}$, such that the polynomials are contained and dense in $E_{n}$ for each $n \in \mathbb{N}$.

Assume that the integration operator $J: E_{n} \rightarrow E_{n}$ is continuous for each $n \in \mathbb{N}$, and that, for each $n \in \mathbb{N}$, every proper closed invariant subspace of $J$ on $E_{n}$ is of the form $A_{K}^{E_{n}}$ for some $K \in \mathbb{N}$. Then

(i) $J: E \rightarrow E$ is continuous, and

(ii) Every proper closed invariant subspace of $J$ on $E$ is of the form

$$
A_{K}^{E}=\left\{f \in E \mid f^{(j)}(0)=0,0 \leq j<K\right\}
$$

for some $K \in \mathbb{N}$. 
Proof. (i) Clearly $J(E) \subset E$ and $J: E \rightarrow E$ is continuous since $E=\operatorname{ind}_{n} E_{n}$ and $J$ is stepwise continuous by assumption.

(ii) The assumptions imply that the polynomials are dense in $E$. The subspaces $A_{K}^{E}$ are proper closed subspaces of $E$ which are invariant for the integration operator $J$.

We fix a proper closed subspace $M$ of $E$ which is invariant for $J$. By Lemma 2.2 there is $n(0) \in \mathbb{N}$ such that $M \cap E_{n}$ is a proper closed subspace of $E_{n}$ for each $n \geq n(0)$. It is also invariant for $J$, since $J(M) \subset M$ and $J\left(E_{n}\right) \subset E_{n}$. By our assumptions, for each $n \geq n(0)$ there is $k(n) \in \mathbb{N}$ such that

$$
M \cap E_{n}=A_{k(n)}^{E_{n}}=\overline{\operatorname{span}\left(\left\{z^{j} \mid j \geq k(n)\right\}\right)} F_{n} .
$$

We select, for each $n \in \mathbb{N}, k(n)$ as the minimum of those $j^{\prime} s$ such that $z^{j} \in M \cap E_{n}$. Since $M \cap E_{n} \subset M \cap E_{n+1}$ for each $n \in \mathbb{N}$, we have $1 \leq k(n+1) \leq k(n) \leq k(n(0))$ for each $n \geq n(0)$. Then there are $L \in \mathbb{N}$ and $n(1) \geq n(0)$ such that $k(n)=L$ for each $n \geq n(1)$. This yields, for each $n \geq n(1)$,

$$
M \cap E_{n}=\left\{f \in E_{n} \mid f^{(j)}(0)=0,0 \leq j<L\right\} .
$$

Therefore

$$
M=\bigcup_{n \geq n(1)}\left(M \cap E_{n}\right)=\left\{f \in E \mid f^{(j)}(0)=0,0 \leq j<L\right\},
$$

and the proof is complete.

\section{Invariant subspaces of the integration operator on Fréchet or (LB)-spaces of holomorphic functions on the disc.}

Let us introduce some notation. We set $R=1$ (for the case of holomorphic functions on $\mathbb{D})$ and $R=+\infty$ (for the case of entire functions). A weight $v$ is a continuous function $v:\left[0, R[\rightarrow] 0, \infty\left[\right.\right.$, which is decreasing on $\left[0, R\left[\right.\right.$ and satisfies $\lim _{r \rightarrow R} r^{n} v(r)=0$ for each $n \in \mathbb{N}$. We extend $v$ radially to $\mathbb{D}$ if $R=1$ and to $\mathbb{C}$ if $R=+\infty$ by $v(z):=v(|z|)$. For such a weight $v$, we define the Banach space $H_{v}^{\infty}$ of holomorphic functions $f$ on the disc $\mathbb{D}$ (if $R=1$ ) or on the whole complex plane $\mathbb{C}$ (if $R=+\infty$ ) such that $\|f\|_{v}:=$ $\sup _{|z|<R} v(z)|f(z)|<\infty$. For a holomorphic function $f \in H(\{z \in \mathbb{C} ;|z|<R\})$ and $r<R$, we denote $M(f, r):=\max \{|f(z)| ;|z|=r\}$. Using the notation $O$ and $o$ of Landau, $f \in H_{v}^{\infty}$ if and only if $M(f, r)=O(1 / v(r)), r \rightarrow R$. It is known that the closure of the polynomials in $H_{v}^{\infty}$ coincides with the Banach space $H_{v}^{0}$ of all those holomorphic functions on $\{z \in \mathbb{C} ;|z|<R\}$ such that $M(f, r)=o(1 / v(r)), r \rightarrow R$, see e.g. [14]. Spaces of type $H_{v}^{\infty}$ appear in the study of growth conditions of holomorphic functions and have been investigated in various articles since the work of Shields and Williams, see e.g. [14, [26] and the references therein. When we must specify the domain of definition of the holomorphic functions, we write $H_{v}^{0}(\mathbb{D})$ or $H_{v}^{0}(\mathbb{C})$.

We recall some examples of weights: For $R=1,(i) v_{\alpha}(r)=(1-r)^{\alpha}$ with $\alpha>$ 0 , which are the standard weights on the disc, $(i i) v(r)=\exp \left(-(1-r)^{-1}\right)$, and (iii) $v(z)=\left(\log \frac{e}{1-r}\right)^{-\alpha}, \alpha>0$. For $R=+\infty,(i) v(r)=\exp \left(-r^{p}\right)$ with $p>0,(i i) v(r)=$ $\exp (-\exp r)$, and $(i i i) v(r)=\exp \left(-\left(\log ^{+} r\right)^{p}\right)$, where $p \geq 2$ and $\log ^{+} r=\max (\log r, 0)$.

A systematic study of inductive and projective limits of weighted Banach spaces of type $H_{v}^{\infty}$ and $H_{v}^{0}$ was initiated by Bierstedt, Meise and Summers in [15]. If $\mathcal{V}=\left(v_{n}\right)_{n}$ is a decreasing sequence of weights on $G=\mathbb{D}$ or $G=\mathbb{C}$, we define the weighted inductive limit by $\mathcal{V}_{0} H(G):=\operatorname{ind}_{n} H_{v_{n}}^{0}(G)$. These (LB)-spaces have been investigated by many authors; 
see e.g. [5], 13] and [14] and the references therein. On the other hand, if $\mathcal{A}=\left(a_{n}\right)_{n}$ is an increasing sequence of weights on $G=\mathbb{D}$ or $G=\mathbb{C}$, the weighted Fréchet space of holomorphic functions on $G$ is defined by $\mathcal{A}_{0} H(G):=\operatorname{proj}_{n} H_{a_{n}}^{0}(G)$. Fréchet spaces of this type were studied by E. Wolf [33]. Concrete examples of spaces of this type in the case of entire functions appear in Section 5. The most relevant examples in the case of holomorphic functions on the disc are Koremblum type spaces, which we define now.

For each $\mu>0$, the growth Banach spaces of holomorphic functions are defined as $A^{-\mu}:=H_{v_{\mu}}^{\infty}$ and $A_{0}^{-\mu}:=H_{v_{\mu}}^{0}$ for the standard weight $v_{\mu}(r)=(1-r)^{\mu}$. These Banach spaces play a relevant role in connection with interpolation and sampling of holomorphic functions; see [23, Section 4.3]. The spaces of holomorphic functions of Korenblum type are defined as follows.

$$
A_{+}^{-\gamma}:=\cap_{\mu>\gamma} A^{-\mu}=\left\{f \in H(\mathbb{D}): \sup _{z \in \mathbb{D}}(1-|z|)^{\mu}|f(z)|<\infty \forall \mu>\gamma\right\},
$$

in which case also

$$
A_{+}^{-\gamma}=\cap_{\mu>\gamma} A_{0}^{-\mu}
$$

for each $\gamma \geq 0$. And

$$
A_{-}^{-\gamma}:=\cup_{\mu<\gamma} A^{-\mu}=\left\{f \in H(\mathbb{D}): \sup _{z \in \mathbb{D}}(1-|z|)^{\mu}|f(z)|<\infty \text { for some } \mu<\gamma\right\}
$$

in which case also

$$
A_{-}^{-\gamma}=\cup_{\mu<\gamma} A_{0}^{-\mu}
$$

for each $0<\gamma \leq \infty$.

The space $A_{+}^{-\gamma}$ is a Fréchet space when endowed with the locally convex topology generated by the increasing sequence of norms $\|f\|_{k}:=\sup _{z \in \mathbb{D}}(1-|z|)^{\gamma+\frac{1}{k}}|f(z)|$, for $f \in$ $A_{+}^{-\gamma}$ and each $k \in \mathbb{N}$. Clearly, $A_{+}^{-\gamma}$ is a Fréchet space of holomorphic functions of type $\mathcal{A}_{0} H(\mathbb{D})$ for an increasing sequence $\mathcal{A}=\left(a_{n}\right)_{n}$.

Each space $A_{-}^{-\gamma}$ is endowed with the finest locally convex topology such that all the natural inclusion maps $A^{-\mu} \subset A_{-}^{-\gamma}$, for $\mu<\gamma$, are continuous. In particular, $A_{-}^{-\gamma}:=$ $\operatorname{ind}_{k} A^{-\left(\gamma-\frac{1}{k}\right)}=\operatorname{ind}_{k} A_{0}^{-\left(\gamma-\frac{1}{k}\right)}$ is an (LB)-space. Of course, the inductive limit is taken over all $k \in \mathbb{N}$ such that $\left(\gamma-\frac{1}{k}\right)>0$. The Korenblum space (see [25]) $A_{-}^{-\infty}$, denoted $A^{-\infty}$, is $A^{-\infty}=\operatorname{ind}_{n} A^{-n}$. All these (LB)-spaces are weighted inductive limits of the form $\mathcal{V}_{0} H(G)$ for a decreasing sequence $\mathcal{V}=\left(v_{n}\right)_{n}$.

Theorem 4.1 (1) If $\mathcal{A}=\left(a_{n}\right)_{n}$ is an increasing sequence of weights on the unit disc $\mathbb{D}$, then the integration operator $J: \mathcal{A}_{0} H(\mathbb{D}) \rightarrow \mathcal{A}_{0} H(\mathbb{D})$ is continuous and every proper closed invariant subspace of $J$ on the weighted Fréchet space $\mathcal{A}_{0} H(\mathbb{D})$ is of the form

$$
\left\{f \in \mathcal{A}_{0} H(\mathbb{D}) \mid f^{(j)}(0)=0,0 \leq j<K\right\}
$$

for some $K \in \mathbb{N}$.

(2) If $\mathcal{V}=\left(v_{n}\right)_{n}$ is a decreasing sequence of weights on the unit disc $\mathbb{D}$, then the integration operator $J: \mathcal{V}_{0} H(\mathbb{D}) \rightarrow \mathcal{V}_{0} H(\mathbb{D})$ is continuous and every proper closed invariant subspace of $J$ on the weighted $(L B)$-space $\mathcal{V}_{0} H(\mathbb{D})$ is of the form

$$
\left\{f \in \mathcal{V}_{0} H(\mathbb{D}) \mid f^{(j)}(0)=0,0 \leq j<K\right\}
$$

for some $K \in \mathbb{N}$. 
Proof. For each weight $v$ on the unit disc $\mathbb{D}$, it follows from 4, proposition 3.1 and Theorem 3.8] (see also [2]), that the integration operator $J: H_{v}^{0}(\mathbb{D}) \rightarrow H_{v}^{0}(\mathbb{D})$ is continuous (even compact), and that every proper closed invariant subspace of $J$ on $H_{v}^{0}(\mathbb{D})$ is of the form

$$
\left\{f \in H_{v}^{0}(\mathbb{D}) \mid f^{(j)}(0)=0,0 \leq j<K\right\}
$$

for some $K \in \mathbb{N}$.

(1) The integration operator on the Fréchet space $\mathcal{A}_{0} H(\mathbb{D})=\operatorname{proj}_{n} H_{a_{n}}^{0}(\mathbb{D})$ satisfies all the hypothesis of Theorem 3.2. The conclusion follows from this result.

$(2)$ Similarly, $J: \mathcal{V}_{0} H(\mathbb{D}) \rightarrow \mathcal{V}_{0} H(\mathbb{D})$ satisfies the assumptions of Theorem 3.3, which permits to complete the proof.

We have the following consequence of Theorem 4.1 ,

Corollary 4.2 The integration operator $J$ is continuous on the Korenblum type spaces $E=A_{+}^{-\gamma}, \gamma \geq 0$ and $E=A_{-}^{-\gamma}, 0<\gamma \leq \infty$, and every proper closed invariant subspace of $J$ on $E$ is of the form $A_{K}^{E}=\left\{f \in E \mid f^{(j)}(0)=0,0 \leq j<K\right\}$ for some $K \in \mathbb{N}$.

\section{Invariant subspaces of the integration operator on Hör- mander algebras.}

A function $p: \mathbb{C} \rightarrow] 0, \infty[$ is called a weight exponent function if it satisfies the following properties: (w1) $p$ is continuous and subharmonic, (w2) $p$ is radial, $p(z)=p(|z|), z \in \mathbb{C}$, (w3) $\log \left(1+|z|^{2}\right)=o(p(z))$ as $|z| \rightarrow \infty$; and (w4) $p$ is doubling, i.e. $p(2 z)=O(p(z)$ ) as $|z| \rightarrow \infty$.

Given a weight $p$, we define the following weighted spaces of entire functions:

$$
A_{p}(\mathbb{C}):=\left\{f \in \mathcal{H}(\mathbb{C}): \text { there is } A>0: \sup _{z \in \mathbb{C}}|f(z)| \exp (-A p(z))<\infty\right\},
$$

endowed with the inductive limit topology, for which it is an (LB)-space and

$$
A_{p}^{0}(\mathbb{C}):=\left\{f \in \mathcal{H}(\mathbb{C}): \text { for all } \varepsilon>0: \sup _{z \in \mathbb{C}}|f(z)| \exp (-\varepsilon p(z))<\infty\right\},
$$

endowed with the projective limit topology, for which it is a Fréchet space.

These spaces are topological algebras. They are called Hörmander algebras of entire functions. Clearly $A_{p}^{0}(\mathbb{C}) \subset A_{p}(\mathbb{C})$. Condition $(w 3)$ implies that $A_{p}^{0}(\mathbb{C})$ contains the polynomials, and condition (w4) implies that the spaces are stable under differentiation. Weighted algebras of entire functions of this type have been considered by many authors. See e.g. [1], [10], 11], 27] and 28] and the references therein.

Here are some examples: When $p(z)=|z|^{s}, s>0$, then $A_{p}(\mathbb{C})$ consists of all entire functions of order $s$ and finite type or order less than $s$; and $A_{p}^{0}(\mathbb{C})$ is the space of all entire functions of order at most $s$ and type 0 . For $s=1, A_{p}(\mathbb{C})$ is the space of all entire functions of exponential type $\operatorname{Exp}(\mathbb{C})$, and $A_{p}^{0}(\mathbb{C})$ is the space of entire functions of infraexponential type.

It was proved by Beltrán, Fernández and the first author in [9, Lemma 4.1] that the integration operator $J$ is continuous on $E=A_{p}(\mathbb{C})$ and $E=A_{p}^{0}(\mathbb{C})$ for every weight exponent $p$. Concerning invariant subspaces we have the following result. 
Theorem 5.1 Let $p$ be a differentiable weight exponent such that $\lim _{r \rightarrow \infty} p^{\prime}(r)=\infty$. Then every proper closed invariant subspace of $J$ on $E=A_{p}^{0}(\mathbb{C})$ or $E=A_{p}(\mathbb{C})$ is of the form

$$
A_{K}^{E}=\left\{f \in E \mid f^{(j)}(0)=0,0 \leq j<K\right\}
$$

for some $K \in \mathbb{N}$. This holds in particular for $p(z)=\alpha|z|^{s}, \alpha>0, s>1$, and for $p(z)=e^{|z|}$.

Proof. Define, for $\beta>0$, the weight $v_{\beta}(z):=\exp (-\beta p(z))$. The polynomials are dense in $H_{v_{\beta}}^{0}$ and, by [3, Theorem 3.8], the operator $J: H_{v_{\beta}}^{0} \rightarrow H_{v_{\beta}}^{0}$ is continuous. Indeed, $w_{\beta}(r):=1 / v_{\beta}(r)=\exp (\beta p(r)), r \geq 0$, satisfies

$$
\liminf _{r \rightarrow \infty} \frac{w^{\prime}(r)}{w(r)}=\liminf _{r \rightarrow \infty} \beta p^{\prime}(r)>1,
$$

since $\lim _{r \rightarrow \infty} \frac{w^{\prime}(r)}{w(r)}=\lim _{r \rightarrow \infty} \beta p^{\prime}(r)=\infty$. Moreover, the latter fact implies by (the proof of) [4, Theorem 3.16] that every proper closed invariant subspace of $J$ on $H_{v_{\beta}}^{0}$ has the form $\left\{f \in H_{v_{\beta}}^{0} \mid f^{(j)}(0)=0,0 \leq j<K\right\}$ for some $K \in \mathbb{N}$.

We have $A_{p}^{0}(\mathbb{C})=\operatorname{proj}_{n} H_{v_{(1 / n)}}^{0}$ and the conclusion follows from Theorem 3.2 since all the hypothesis of this result hold.

Similarly, since $A_{p}(\mathbb{C})=\operatorname{ind}_{n} H_{v_{n}}^{0}$ the result follows from Theorem 3.3 in this case.

A different approach enables us to treat the case of the integration operator on the $(\mathrm{LB})$-algebra $\operatorname{Exp}(\mathbb{C})$ of entire functions of exponential type.

Let $(B f)(z)=\frac{f(z)-f(0)}{z}$ denote the backward shift on $H(\mathbb{C})$. We recall that the norm in the Hardy space $H^{2}$ is given by

$$
\|f\|_{H^{2}}=\sup _{0 \leq r<1}\left(\frac{1}{2 \pi} \int_{0}^{2 \pi}\left|f\left(r e^{i t}\right)\right|^{2} d t\right)^{\frac{1}{2}} .
$$

In what follows we denote

$$
g_{\varepsilon}(z):=g(\varepsilon z)
$$

Lemma 5.2 Any transcendent function $f \in H(\mathbb{C})$ is a cyclic vector for $B$.

Proof. According to [21, Theorem 2.2.4], the restriction of any transcendent function to the unit circle is a cyclic vector for the backward shift on the Hardy space $H^{2}$. We now fix a compact set $K \subset \mathbb{C}$ and $g \in H(\mathbb{C})$. Take $r>0$ so that $|z| \leq r$ for all $z \in K$ and put $R=2 r$. Since

$$
B^{n}\left(f_{R}\right)=R^{n}\left(B^{n} f\right)_{R}
$$

we have

$$
g_{R} \in \overline{\operatorname{span}}\left(\left(B^{n} f\right)_{R}: n \in \mathbb{N}\right),
$$

where the closure is taken in $H^{2}$. So, for every $\varepsilon>0$ we can find coefficients $a_{1}, \ldots a_{N}$ such that

$$
\left.\| g_{R}-\sum_{k=1}^{N} a_{k}\left(B^{k} f\right)_{R}\right) \|_{H^{2}}<\varepsilon .
$$


For every $z \in K$ we have

$$
\begin{aligned}
\left|g(z)-\sum_{k=1}^{N} a_{k}\left(B^{k} f\right)(z)\right| & \leq \frac{R}{2 \pi} \int_{0}^{2 \pi} \frac{\left|g\left(R e^{i t}\right)-\sum_{k=1}^{N} a_{k}\left(B^{k} f\right)\left(R e^{i t}\right)\right|}{\left|R e^{i t}-z\right|} d t \\
& \leq \frac{2}{2 \pi} \int_{0}^{2 \pi}\left|g\left(R e^{i t}\right)-\sum_{k=1}^{N} a_{k}\left(B^{k} f\right)\left(R e^{i t}\right)\right| d t \\
& \left.\leq 2 \| g_{R}-\sum_{k=1}^{N} a_{k}\left(B^{k} f\right)_{R}\right) \|_{H^{2}}<2 \varepsilon .
\end{aligned}
$$

Theorem 5.3 Every proper closed invariant subspace of $J$ on $E=E x p(\mathbb{C})$ has the form $A_{K}^{E}=\left\{f \in E: f^{j}(0)=0,0 \leq j<K\right\}$ for some $K \in \mathbb{N}$.

Proof. We consider the topological isomorphism (see for instance [31, p.94])

$$
\Phi: \operatorname{Exp}(\mathbb{C}) \rightarrow H(\mathbb{C})_{b}^{\prime},\langle\Phi(g), f\rangle=\sum_{k=0}^{\infty} a_{k} b_{k} k !
$$

where

$$
f(z)=\sum_{k=0}^{\infty} a_{k} z^{k}, g(z)=\sum_{k=0}^{\infty} b_{k} z^{k}
$$

We identify $f \in H(\mathbb{C})$ with $T_{f} \in H(\mathbb{C})^{\prime \prime}, T_{f}(u):=u(f)$. Then

$$
\Phi^{t}: H(\mathbb{C}) \rightarrow \operatorname{Exp}(\mathbb{C})^{\prime},\left\langle\Phi^{t}\left(T_{f}\right), g\right\rangle=\left\langle T_{f}, \Phi(g)\right\rangle=\Phi(g)(f) .
$$

Let us assume that $M$ is a proper closed subspace of $\operatorname{Exp}(\mathbb{C})$ satisfying $J(M) \subset M$. Obviously we also have $J^{t}\left(M^{\circ}\right) \subset M^{\circ}$. We now check that

$$
J^{t} \circ \Phi^{t}=\Phi^{t} \circ B .
$$

For $f \in H(\mathbb{C}), f(z)=\sum_{k=0}^{\infty} a_{k} z^{k}$ and $g \in \operatorname{Exp}(\mathbb{C}), g(z)=\sum_{k=0}^{\infty} b_{k} z^{k}$, we have

$$
\left\langle\left(J^{t} \circ \Phi^{t}\right)(f), g\right\rangle=\langle f, \Phi(J g)\rangle=\sum_{k=1}^{\infty} a_{k} \frac{b_{k-1}}{k} k !=\sum_{k=1}^{\infty} a_{k} b_{k-1}(k-1) ! .
$$

Also

$$
\left\langle\left(\Phi^{t} \circ B\right)(f), g\right\rangle=\langle B(f), \Phi(g)\rangle=\sum_{k=0}^{\infty} a_{k+1} b_{k} k ! .
$$

Identity (5.1) is proved. Consequently

$$
F:=\left(\Phi^{t}\right)^{-1}\left(M^{\circ}\right)
$$

is a proper closed subspace of $H(\mathbb{C})$ and satisfies $B(F) \subset F$. According to Lemma [5.2, $F$ cannot contain transcendent functions. Since $F$ is a closed subspace of the Fréchet space $H(\mathbb{C})$ and it consists only of polynomials then an application of Baire's theorem permits us to conclude that $F$ is finite dimensional. Let $f_{0}(z)=\sum_{k=0}^{N-1} a_{k} z^{k}+z^{N}$ be an element of 
$F$ with the largest possible degree $N$. We observe that $\left(B f_{0}\right)(z)=\sum_{k=0}^{N-2} a_{k+1} z^{k}+z^{N-1}$. Then the polynomials

$$
f_{0}, B f_{0}, B^{2} f_{0}, \ldots, B^{N} f_{0}
$$

are linearly independent and we conclude

$$
\operatorname{span}\left\{f_{0}, B f_{0}, B^{2} f_{0}, \ldots, B^{N} f_{0}\right\}=\operatorname{span}\left\{1, z, z^{2}, \ldots, z^{N}\right\}=F .
$$

Finally

$$
\begin{aligned}
M=M^{\circ \circ} & =\left\{g \in \operatorname{Exp}(\mathbb{C}) \mid\left\langle\Phi^{t}(f), g\right\rangle=0 \forall f \in F\right\} \\
& =\left\{g \in \operatorname{Exp}(\mathbb{C}) \mid\left\langle z^{k}, \Phi(g)\right\rangle=0,0 \leq k \leq N\right\} \\
& =\left\{g \in \operatorname{Exp}(\mathbb{C}) \mid g^{(k)}(0)=0,0 \leq k \leq N\right\}
\end{aligned}
$$

Remark 5.4 The integration operator is continuous on all the Hörmander algebras defined above $A_{p}^{0}(\mathbb{C})$ and $A_{p}(\mathbb{C})$ by $[9$, Lemma 4.1]. However, we do not know whether the conclusion of Theorem 5.1 holds if the condition $\lim _{r \rightarrow \infty} p^{\prime}(r)=\infty$ fails, in particular for the weight exponents $p(z)=|z|^{s}, 0<s \leq 1$, except in the case covered by Theorem 5.3.

Acknowledgement. This research was partially supported by the projects MTM201676647-P and GV Prometeo/2017/102.

\section{References}

[1] A.V. Abanin, Effective and sampling sets for Hörmander spaces, Complex Anal. Oper. Theory 12 (2018), no. 6, 1401-1419.

[2] A.V. Abanin, P.T. Tien, The algebraic equalities and their topological consequences in weighted spaces. J. Math. Anal. Appl. 422 (2015), no. 1, 435-445.

[3] A.V. Abanin, P.T. Tien, Differentiation and integration operators on weighted Banach spaces of holomorphic functions. Math. Nachr. 290 (2017), no. 8-9, 1144-1162.

[4] A.V. Abanin, P.T. Tien, Invariant subspaces for classical operators on weighted spaces of holomorphic functions. Integral Equations Operator Theory 89 (2017), no. 3, 409438.

[5] A.V. Abanin, P.T. Tien, Compactness of classical operators on weighted Banach spaces of holomorphic functions. Collect. Math. 69 (2018), no. 1, 1-15.

[6] A.A. Albanese, J. Bonet, W.J. Ricker, The Cesàro operator on Korenblum type spaces of analytic functions, Collect. Math. 69 (2018), 263-281.

[7] A. Aleman, B. Korenblum, Volterra invariant subspaces of $H^{p}$, Bull. Sci. Math. 132 (2008), 510-528.

[8] M.J. Beltrán, J. Bonet, C. Fernández, Classical operators on weighted Banach spaces of entire functions. Proc. Amer. Math. Soc. 141 (2013), no. 12, 4293-4303. 
[9] M.J. Beltrán, J. Bonet, C. Fernández, Classical operators on the Hörmander algebras. Discrete Contin. Dyn. Syst. 35 (2015), no. 2, 637-652.

[10] C.A. Berenstein, R. Gay, Complex Analysis and Special Topics in Harmonic Analysis, Springer, New York, 1995.

[11] C.A. Berenstein, B.Q. Li, A. Vidras, Geometric characterization of interpolating varieties for the (FN)-space $A_{p}^{0}$ of entire functions, Can. J. Math. 47 (1995), 28-43.

[12] K.D. Bierstedt, J. Bonet, Some aspects of the modern theory of Fréchet spaces, RACSAM Rev. R. Acad. Cien. Serie A. Mat. 97 (2003), 159-188.

[13] K.D. Bierstedt, J. Bonet, Weighted (LB)-spaces of holomorphic functions: $V H(G)=$ $V_{0} H(G)$ and completeness of $V_{0} H(G)$, J. Math. Anal. Appl. 323 (2006), 747-767.

[14] K.D. Bierstedt, J. Bonet, A. Galbis, Weighted spaces of holomorphic functions on bounded domains, Michigan Math. J. 40 (1993), 271-297.

[15] K.D. Bierstedt, R. Meise, W.H. Summers, A projective description of weighted inductive limits. Trans. Amer. Math. Soc. 272 (1982), no. 1, 107-160.

[16] J. Bonet, Invariant subspaces for the integration operators on weighted locally convex spaces of holomorphic functions, Arvix 2003.13573 (2020).

[17] J. Bonet, C. Fernández, The range of the restriction map for a multiplicity variety in Hörmander algebras of entire functions. Mediterr. J. Math. 11 (2014), no. 2, 643-652.

[18] J. Bonet, W. Lusky, J. Taskinen, Monomial basis in Korenblum type spaces of analytic functions, Proc. Amer. Math. Soc. 146 (2018), no. 12, 5269-5278.

[19] O. Constantin, A Volterra-type integration operator on Fock spaces, Proc. Amer. Math. Soc. 140 (2012), 4247-4257.

[20] O. Constantin, J.A. Peláez, Integral operators, embedding theorems and a LittlewoodPaley formula on weighted Fock spaces, J. Geom. Anal. 26 (2016), 1109-1154.

[21] R.G. Douglas, H.S. Shapiro, A.L. Shields, Cyclic vectors and invariant subspaces for the backward shift operator, Ann. Inst. Fourier 20 (1970), 37-76.

$[22]$ A. Harutyunyan, W. Lusky, On the boundedness of the differentiation operator between weighted spaces of holomorphic functions. Studia Math. 184 (2008), no. 3, 233-247.

[23] H. Hedenmalm, B. Korenblum, K. Zhu, Theory of Bergman Spaces. Grad. Texts in Math. 199, Springer-Verlag, New York, 2000.

[24] H. Jarchow, Locally Convex Spaces, Teubner, Stuttgart, 1981.

[25] B. Korenblum, An extension of the Nevanlinna theory, Acta Math. 135 (1975), 187219.

[26] W. Lusky, On the isomorphism classes of weighted spaces of harmonic and holomorphic functions, Studia Math. 175 (2006), 19-45.

[27] R. Meise, Sequence space representations for (DFN)-algebras of entire functions modulo closed ideals, J. Reine Angew. Math. 363 (1985), 59-95. 
[28] R. Meise, B.A. Taylor, Sequence space representations for (FN)-algebras of entire functions modulo closed ideals, Studia Math. 85 (1987), 203-227.

[29] R. Meise and D. Vogt. Introduction to Functional Analysis, Clarendon Press Oxford University Press, New York, 1997.

[30] W. Rudin, Real and Complex Analysis, Mc Grawn Hill, New York, 1974.

[31] F. Trèves, Locally convex spaces and linear partial differential equations. Die Grundlehren der mathematischen Wissenschaften 146, Springer-Verlag, New York 1967.

[32] D. Vogt, Non-natural topologies on spaces of holomorphic functions. Ann. Polon. Math. 108 (2013), no. 3, 215-217.

[33] E. Wolf, Weighted Fréchet spaces of holomorphic functions, Studia Math. 174 (2006), no. $3,255-275$.

[34] K. Zhu, Operator Theory on Function Spaces, Math. Surveys and Monographs Vo. 138. Amer. Math. Soc. 2007.

[35] K. Zhu, Analysis on Fock spaces, Graduate Texts in Mathematics, 263. Springer, New York, 2012.

\section{Authors' address:}

José Bonet: Instituto Universitario de Matemática Pura y Aplicada IUMPA, Universitat Politècnica de València, E-46071 Valencia, Spain

email: jbonet@mat.upv.es

Antonio Galbis: Departament d'Anàlisi Matemàtica, Universitat de València, E-46100

Burjassot (València), Spain

email: antonio.galbis@uv.es 